\title{
IN-SITU EXPLORATION OF DUST IN THE SOLAR SYSTEM AND INITIAL RESUITS FROM THE GALILEO DUST DETECTOR
}

\author{
E. GRU̇N ${ }^{1}$, H. FECHTIG ${ }^{1}$, M. S. HANNER ${ }^{2}$, J. KISSEL ${ }^{1}$, \\ B.-A. LINDBLAD ${ }^{3}$, D. LINKERT ${ }^{1}$, G. MORFILL ${ }^{4}$ and H. A. ZOOK \\ 1 Max-Plank-Institut für Kernphysik, 6900 Heidelberg, Germany \\ 2 Jet Propulsion Laboratory, Pasadena, CA 91103, U.S.A. \\ 3 Lund Observatory, 221 Lund, Sweden \\ 4 Max-Planck-Institut für Extraterrestrische Physik, \\ 8046 Garching, Germany \\ 5 NASA Johnson Space Center, Houston, TX 77058, U.S.A.
}

\begin{abstract}
In-situ measurements of interplanetary dust have been performed in the heliocentric distance range from $0.3 \mathrm{AU}$ out to $18 \mathrm{AU}$. Due to their small sensitive areas (typically $0.01 \mathrm{~m}^{2}$ for the highly sensitive impact ionization sensors) or low mass sensitivities $\left(210^{-9} \mathrm{~g}\right.$ of the large area penetration detectors) previous instruments recorded only a few 100 impacts during their lifetimes. Nevertheless, important information on the distribution of dust in interplanetary space has been obtained between 0.3 and 18 AU distance from the Sun. The Galileo dust detector combines the high mass sensitivity of impact ionization detectors $\left(10^{-15} \mathrm{~g}\right)$ together with a large sensitive area $\left(0.1 \mathrm{~m}^{2}\right)$. The Galileo spacecraft was launched on October 18 , 1989 and is on its solar system cruise towards Jupiter. Initial measurements of the dust flux from 0.7 to $1.2 \mathrm{AU}$ are presented.
\end{abstract}

\section{Introduction}

There are several methods to study various aspects of interplanetary dust from the Earth. Observations of the scattered light (zodiacal light) from interplanetary dust and its thermal emission reveal the large scale spatial distribution of particles in the $10 \mu \mathrm{m}$ to 1 millimeter size range. Meteor observations refer to $\mathrm{mm}$ and larger objects, the orbits of which intersect the Earth. Interplanetary dust particles collected in the stratosphere allow us to obtain compositional and morphological information. From lunar microcrater studies, the size distribution of sub micron to $\mathrm{mm}$ sized particles was determined (for a review see Leinert and Grün, 1990).

Complementary to the above mentioned methods are in-situ studies by dust impact detectors on board interplanetary spacecraft. The purpose of this paper is to give a survey of previous interplanetary dust instruments and their major findings, and to present first results of the dust detector on board the Galileo spacecraft.

A.C. Levasseur-Regourd and H. Hasegawa (eds.), Origin and Evolution of Interplanetary Dust, 21-28.

- 1991 Kluwer Academic Publishers, Printed in Japan. 


\section{Characteristics of Recent In-Situ Dust Detectors.}

In-situ measurements of interplanetary dust have been performed in the heliocentric distance range from $0.3 \mathrm{AU}$ out to $18 \mathrm{AU}$ (Table 1). We have included in the list of interplanetary dust detectors also the two Earth satellites HEOS 2 (Hoffmann et al., 1975) and Hiten (Igenbergs et al., 1990a) because they performed significant portions of their measurements outside the range of influence of the Earth.

Two types of impact detectors were used for interplanetary dust measurements: impact ionization detectors with detection thresholds of $10^{-16}$ to $10^{-13} \mathrm{~g}$ and penetration detectors with detection thresholds of $10^{-9}$ and $10^{-8} \mathrm{~g}$. These detection thresholds refer to a typical impact speed of 20 $\mathrm{km} / \mathrm{s}$. The penetration detectors on board Pioneers 10 and 11 (Humes, 1980) have large geometric factors, i.e. sensitive areas and effective solid angles (for reference, a flat plate has $\pi \mathrm{sr}$ effective solid angle). Most impact ionization detectors have sensitive areas of only $0.01 \mathrm{~m}^{2}$, except the Galileo instrument (Grün et al., 1990) which has a ten times larger sensitive area. Because the effective solid angles of the HEOS 2, Helios $1 / 2$ (Dietzel et al., 1973) and Galileo detectors are significantly less than that for a flat plate, they are able to provide better directional information. E.g. in an isotropic flux half of the particles are recorded within a cone of 32 degrees half angle around the axis of the Galileo sensor. The Pioneer 8 and 9 detectors (Berg and Richardson, 1969) could record approximate directions for a few time-of-flight events, however, most of the impacts were recorded by the wide angle front film sensor (Berg and Grün, 1973).

The dynamic range of the instruments describes the range over which particle masses can be determined at a given impact speed. For larger particles the instrument reaches saturation and only lower mass limits can be stated. Dynamic range of 1 for the Pioneer 10 and 11 instruments implies that only lower mass limits for all impacts can be stated. The last two columns of table 1 show theoretical performance parameters of the different

TABLE 1: In situ dust detectors in interplanetary space
(1) heliocentric distançe (AU)
(3) sensitive areae $\left(\mathrm{m}^{2}\right)$
(2) mass threshold at $20 \mathrm{~km} / \mathrm{s}(\mathrm{g})$
(5) dynamic range
(4) effective solid angle (sr)
(7) largest particle in $1 \mathrm{yr}$ at $1 \mathrm{AU}(\mathrm{g})$

\begin{tabular}{lclllllll}
\hline Spacecraft & $(1)$ & $(2)$ & $(3)$ & $(4)$ & $(5)$ & $(6)$ & $(7)$ \\
\hline Pioneer 8 & $0.97-1.09$ & 2 & $10^{-13}$ & 0.0094 & 2.9 & 200 & 17 & $510^{-10}$ \\
Pioneer 9 & $0.75-0.99$ & 2 & $10^{-13}$ & 0.0074 & 2.9 & 200 & 14 & $310^{-10}$ \\
HEOS 2 & 1 & 2 & $10^{-16}$ & 0.01 & 1.03 & $10^{4}$ & 370 & $310^{-11}$ \\
Pioneer 10 & $1-18$ & 2 & $10^{-9}$ & $0.26^{*}$ & $3^{* *}$ & 1 & 19 & $310^{-7}$ \\
Pioneer 11 & $1-10$ & 1 & $10^{-8}$ & $0.26^{*}$ & $3^{* *}$ & 1 & 10 & $310^{-7}$ \\
Helios 1/2 & $0.3-1$ & 9 & $10^{-15}$ & 0.012 & 1.23 & $10^{4}$ & 40 & $910^{-11}$ \\
Galileo & $0.7-5.2$ & 1 & $10^{-15}$ & 0.1 & 1.4 & $10^{6}$ & 1500 & $210^{-8}$ \\
Hiten & 1 & 1 & $10^{-15}$ & 0.01 & $2^{* *}$ & $310^{4}$ & 220 & $210^{-10}$ \\
\hline
\end{tabular}

* initial area, actual area decreased as cells were punctured

** estimated values 


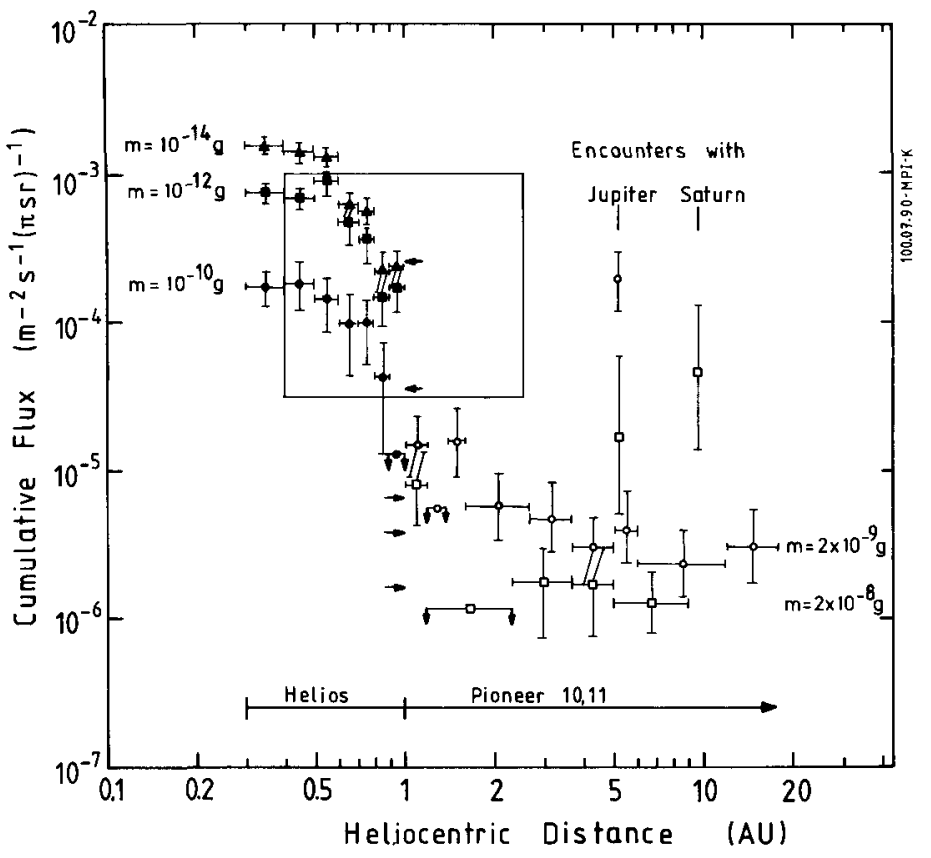

Figure 1. Flux of interplanetary dust particles measured by the Helios and Pioneer 10 and 11 spaceprobes. The different symbols represent flux values for five different mass thresholds. Zero impacts recorded in specific distance intervals are presented by upper flux limits. For comparison corresponding fluxes determined at $1 \mathrm{AU}$ are indicated by horizontal arrows. The rectangular insert shows the ordinate and abscissa ranges used in Figure 4 where the Galileo data are displayed.

instruments. For the interplanetary dust flux at 1 AU given by Grün et al. (1985b) the following values are calculated: column 6, the number of impacts per year above the detection threshold and column 7, the largest particle that would be recorded in one year. In these calculations only the geometric factors have been considered but not the orbits of the spacecraft and the actual orientations of the sensors with respect to the Sun and the ecliptic plane. Therefore, the actually measured quantities may deviate somewhat from the given values.

\section{Interplanetary Dust Measurements}

In this section we describe major results from the various interplanetary dust measurements. A radial profile of the dust flux in the inner solar system between 1 and 0.3 AU distance from the Sun has been determined by the Helios 1 and 2 spaceprobes (Grin et al., 1985a). Figure 1 shows radial flux profiles for 3 threshold masses adjusted for a flat plate sensor. Helios measurements allowed us to identify two dynamically different interplanetary 
dust populations in the inner solar system (Grün et al., 1980): (1) particles which orbit the sun on low eccentric orbits $(e<0.4)$ with rather small semi major axes ( $a \leq 0.5 \mathrm{AU}$ ) - this population had already been noticed by the previous Pioneer 8/9 (Berg and Grïn, 1973) and HEOS 2 (Hoffmann et al., 1975) dust experiments - and (2) particles on highly eccentric orbits (e > 0.4) which have also large semi major axes (a > 0.5 AU).

The Pioneer 8 and 9 experiments detected a dominant flux of small particles from approximately the solar direction. Existence of these particles was recently confirmed by Hiten (Igenbergs et al., 1990b) measurements. A radial profile of the dust flux (Zook, 1975) could not conclusively be determined from the Pioneer $8 / 9$ measurements since McDonnell et al. (1975) could explain the same data as variation with heliocentric longitude. With the Pioneer 8/9 and HEOS 2 measurements it was possible to determine the flux of small $\left(\leq 10^{-12} \mathrm{~g}\right)$ interplanetary particles at $1 \mathrm{AU}$ (cf. Grün et al., 1985).

In the outer solar system the penetration detectors on board Pioneers 10 and 11 determined the interplanetary dust flux (Humes, 1980). Figure 1 shows radial flux profiles for the two threshold masses. No sign of an increase in the asteroid belt was detected and outside about $3 \mathrm{AU}$ they recorded a flat flux profile, except for strong increases of the flux near Jupiter and Saturn. The Pioneer 11 data obtained between 4 and 5 AU are best explained by meteoroids moving on highly eccentric orbits.

For comparison cumulative flux values measured by detectors in Earth-like orbits are shown in Figure 1 for the same five mass thresholds. The obvious discrepancy of the fluxes at 1 AU can not be explained only by the large statistical uncertainties of the spaceprobe measurements. The measurements presented were obtained by detectors which moved on different heliocentric trajectories at $1 \mathrm{AU}$. Therefore, the different fluxes measured reflect different relative speeds with respect to the interplanetary dust cloud. Other differences are the viewing directions of the sensors which have an influence as well since the flux is not isotropic. These considerations make it difficult to determine the radial profile of the spatial density of dust at $1 \mathrm{AU}$.

\section{The Gallleo Dust Experiment}

The Galileo spacecraft was launched on 18 October 1989 on its trajectory to Jupiter. However, before the Galileo spacecraft reaches Jupiter it has to perform a six year journey through the solar system before it will become the first man made satellite of the giant planet. Figure 2 shows the first portion of the Galileo trajectory through the inner solar system. A Venus swing by took place on 8 . February 1990 and the spacecraft flew by the Earth in December 1990 for the first time.

The dust detector instrument is mounted to the spinning section of the spacecraft. During this initial phase of the mission the spin vector points away from the Sun. The viewing direction of the dust detector forms an angle of 55 degrees with the spin vector. This angle has been chosen for optimum dust detection geometry at Jupiter. The Galileo dust instrument is a multi-coincidence impact ionization detector (Grün et al., 1990), with geometric factors given in Table 1. From the released impact charge and the time relations of different charge signals, the mass, speed and electric charge of impacting dust particles can be determined. From each impact or noise 


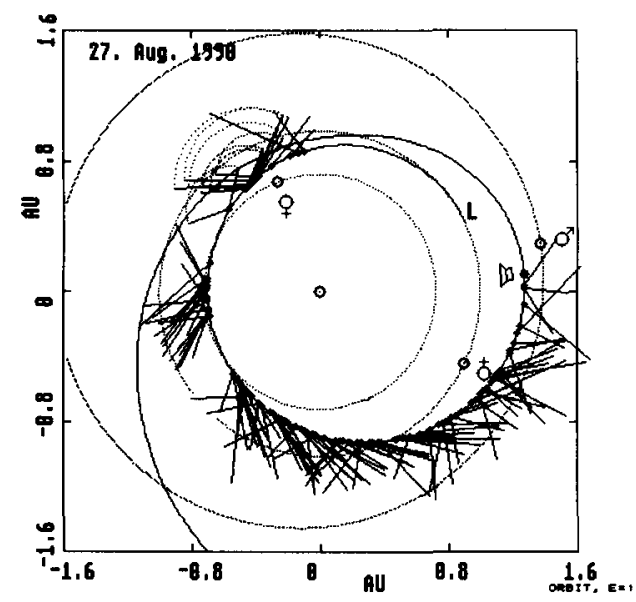

Figure 2. View onto the ecliptic plane with the orbits of Venus, Earth, Mars and the Galileo spacecraft. Launch (L) took place on 18. October 1989. The positions of the planets and Galileo are shown for the given date. Discrete dust impacts are indicated by dots on the Galileo orbit and the corresponding sensor pointing directions at impact (projection onto the ecliptic plane) are give by the attached bars. The length of the bars indicate the magnitude of the impact charge. A fan presents the full range of sensor pointing directions for a time when no pointing information was available.

event all the measured quantities are transmitted to ground and each event is counted in one of several accumulators.

\section{Flrst Results}

On 28. December 1989 the dust detector was switched on for the first time, when Galileo was at a distance of $0.88 \mathrm{AU}$ from the Sun. During a three day configuration period the instrument was set to a state where noise was sufficiently suppressed and impact events could be reliably distinguished from noise events. Around the time of the Venus fly-by an increased noise rate was encountered, so that the sensitivity threshold of the instrument had to be increased in order to cut the noise down to $\leq 5$ events per day.

The recorded discrete impacts are displayed in Figure 2. Dust impacts are overlaid on Galileo's orbit. The impact direction is indicated as well as the magnitude of the impact. During most of the time data were transmitted in small packets only once or twice a week. Apparent gaps in the distribution of impacts are due to two approximately 4-weeks long gaps in data transmission. Therefore, information on discrete impacts is only complete to $55 \%$. However, from the accumulated data the impact rate can be reconstructed over the whole period without any gaps (Figure 3). The highest short-term rate of 3.5 impacts per day was observed shortly after perihelion passage. The impact rate presented here corresponds to an operational mass threshold which is approximately a factor of 10 above sensitivity threshold 


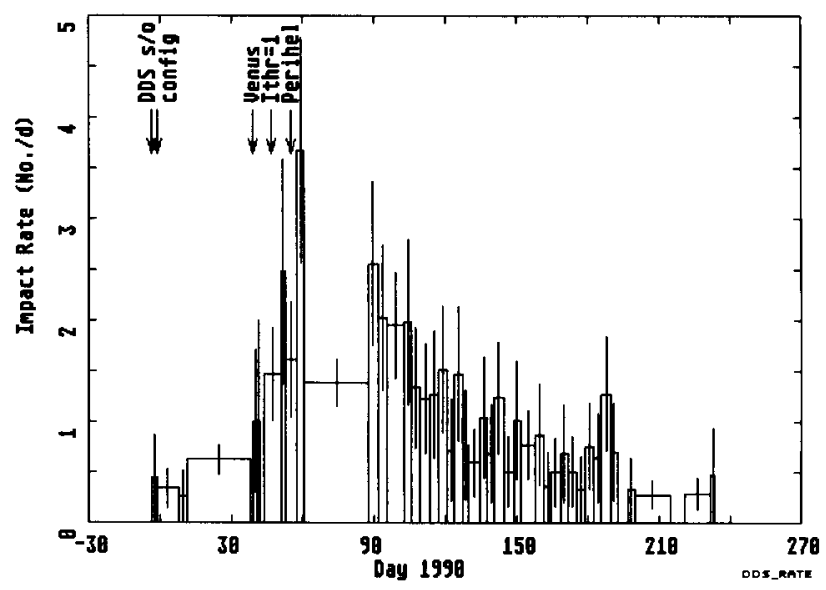

Figure 3. Impact rate observed by the Galileo dust detector until 27. August 1990. Major events are indicated: instrument switch-on (DDS s/o), termination of instrument configuration (config), Venus fly-by (Venus), change of the sensitivity threshold to suppress excessive noise (Ithr=1) and perihelion passage (Perihel).

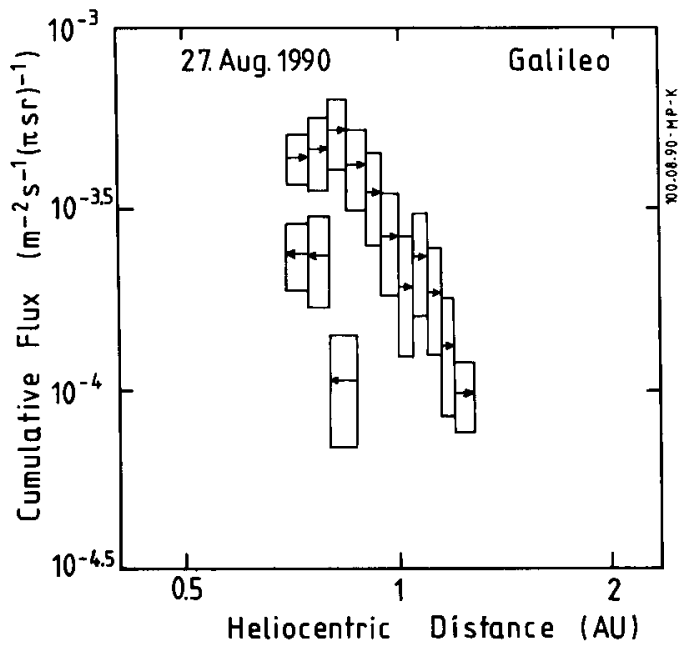

Figure 4. Cumulative flux of dust particles recorded by the Galileo dust detector until the indicated date. The flux corresponds to an operational mass threshold of $10^{-14} \mathrm{~g}$ (for an assumed impact speed of $20 \mathrm{~km} / \mathrm{s}$ ) and to a flat plate sensor (cf. Figure 1). The arrows represent the averaged fluxes and indicate whether Galileo was moving inward or outward. Each box represents the distance interval over which the flux was averaged and the 1-o uncertainty of this flux. 
given in Table 1. The full sensitivity will only be reached when higher a data transmission rate can be established in mid 1991.

In order to allow the comparison with other flux data we have rebinned the data in about equal sized distance intervals and calculated the flux onto a flat plate sensor by assuming an isotropic flux. Figure 4 shows the measured flux during the initial Galileo mission phase. The general decrease of the flux with heliocentric distance is due to the decrease of the dust population with increasing distance from the sun (cf. Figure 1). No significant deviation from a smooth slope at $1 \mathrm{AU}$ is observed. The inbound fluxes are factors two to five smaller than the outbound fluxes at the same heliocentric distance. This can be understood by the orientation of the viewing cone of the dust sensor relative to the orbit of the spacecraft. In the outbound section the fluxes are comparatively higher because the spacecraft motion is outward towards the hemisphere the sensor is facing. A first analysis of these data indicates that the dust population observed by Galileo is compatible with dust particles on low eccentric $(e \leq 0.3)$ orbits.

ACKNOWLEDGEMENTS. The authors are especially indebted to G. Linkert, D. Maas, G. Matt, G. McSmith, C. Polanskey and N. Siddique for their personal involvement during the initial phases of the Galileo mission. This work has been supported by the Bundesminister für Forschung and Technologie, under the grant 01 QJ 900400.

\section{References}

Berg, O. E. and Grün, E. (1973) Evidence of hyperbolic cosmic dust particles. Space Research XIII, 1047-1055.

Berg, O. E. and Richardson, F. F. (1986) The Pioneer 8 cosmic dust experiment, Rev. Sci. Instrum. 40, 1333-1337.

Dietzel, H., Eichhorn, G., Fechtig, H., Gruin, E., Hoffmann, H. J. und Kissel, J. (1973) The HEOS 2 and Helios micrometeoroid experiments, J. Phys. (E) Scientific Instrum., 6, 209-217.

Grün, E., Pailer, N., Fechtig, H. and Kissel, J. (1980) Orbital and physical characteristics of micrometeoroids in the inner solar system as observed by Helios 1, Planet. Space Sci., 29, 333-349.

Gruin, E., Fechtig, H. and Kissel, J. (1985a) Orbits of interplanetary dust particles inside $1 \mathrm{AU}$ as observed by Helios, in Properties and Interactions of Interplanetary Dust, R. H. Giese and P. Lamy (eds.), Reidel, Dordrecht, pp. 105-111.

Grün, E., Zook, H. A., Fechtig, H., and Giese, R. H. (1985b) Collisional balance of the meteoritic complex, Icarus, 62, 244-272.

Grün, E., Fechtig, H., Hanner, M. S., Kissel, J., Lindblad, B. A., Linkert, D., Morfill, G. E. and Zook, H. A. (1990) The Galileo dust detector, submitted to Space Sci. Rev.

Hoffmann, H. J., Fechtig, H., Grün, E. und Kissel, J. (1975) Temporal fluctuation and anisotropy of the micrometeoroid flux in the earth-moon system, Plant. Space Sci., 23, 985-991.

Humes, D. H. (1980) Results of Pioneer 10 and 11 meteoroid experiments: Interplanetary and near-Saturn. Journ. Geophys. Res., 85, 5841-5852. 
Igenbergs, E., Huidepohl, A., Uesugi, K. T., Hayashi, T., Svedham, H., Igelseder, H., Koller, G., Glasmachers, A., Grün, E., Schwehm, G., Mizutani, H., Yamamoto, T., Fujimura A., Ishii, N., Yamakoshi, K. and Nogami, K. (1990a) The Munich dust counter - A cosmic dust experiment on board of the Muses-A mission of Japan, in Origin and Evolution of Interplanetary Dust, This issue.

Igenbergs, E., Hüdepohl, A., Uesugi, K. T., Hayashi, T., Svedham, H., Igelseder, H., Koller, G., Glasmachers, A., Grün, E., Schwehm, G., Mizutani, H., Yamamoto, T., Fujimura A., Ishii, N., Yamakoshi, K. and Nogami, K. (1990b) The present status of the Munich dust counter experiment on board of the Hiten spacecraft, in Origin and Evolution of Interplanetary Dust, This issue.

Leinert, C. and Griin, E. (1990) Interplanetary dust, in Physics of the Inner Heliosphere, R. Schwenn and E. Marsch (eds.), Springer-Verlag, Heidelberg, pp. 207-275

McDonnell, J. A. M., Berg, O. E., and Richardson, F. F. (1975) Spatial and time variations of the interplanetary microparticle flux analyzed from deep space probes Pioneers 8 and 9, Planet. Space Sci. 23, 205-214.

Zook, H. A. (1975) Hyperbolic cosmic dust: its origin and its astrophysical significance, Planet. Space Sci. 23, 1391-1397. 